\title{
In Silico Studies on Development of Novel Virostatic Agents against Bluetongue Virus
}

\author{
Pandrangi Anupama \\ Department of Chemistry, Nizam College, Basheerbagh, Hyderabad 500 001, India \\ Correspondence should be addressed to Pandrangi Anupama; p_anupama2002@yahoo.com
}

Received 9 December 2013; Accepted 15 January 2014; Published 4 March 2014

Academic Editors: J. Ortego and J. Stephenson

Copyright (C) 2014 Pandrangi Anupama. This is an open access article distributed under the Creative Commons Attribution License, which permits unrestricted use, distribution, and reproduction in any medium, provided the original work is properly cited.

\begin{abstract}
The core of BTV is organized into three concentric structures of which VP7 protein forms the major core protein. The subcore consists of VP3 protein and the innermost part of the core is made of three minor proteins: VP1, VP4, and VP6. Earlier it was reported that core-like particles (CLPs) composed of viral VP7 and VP3 proteins were produced in order to study role of VP7 protein in intermolecular interactions in the BTV assembly process. Site specific mutational studies revealed that substitution of the single lysine residue of VP7 (Lys-255) by leucine abrogated CLP formation, indicating a critical role for this lysine. In the present study, homology modeling, mutagenesis, and docking studies were carried out in order to design potent leads in modulation of VP7 protein in abrogating CLP formation.
\end{abstract}

\section{Introduction}

Bluetongue is an infectious noncontagious virus disease of ruminants caused by bluetongue virus of genus Orbivirus within the family Reoviridae. It is transmitted by biting midges of the Culicoides genus (Diptera: Ceratopogonidae). In addition to biting midges, BTV has been isolated from some arthropods, for example, sheep ked (Melophagus ovinus) [1] or some species of ticks [2,3] and mosquitoes [4]. However, these are mechanical vectors with only a negligible role in disease epidemiology [5]. It can be directly transmitted from one animal to another through semen and transplacentally [6]. Bluetongue can also be spread by live attenuated vaccines against BTV or even by vaccines against other antigens contaminated with BTV $[7,8]$.

To date 26 distinct internationally recognized serotypes (based on the lack of cross-neutralization) of the virus have been identified. Cattle and goats are major hosts of the virus, but in these species infection is usually asymptomatic despite high virus levels, allowing the disease to circulate in the absence of any symptoms. Sheep and deer are usually the only species to exhibit symptoms of infection. The manifestations of bluetongue range from an unapparent to a fatal outcome depending on the serotype and strain of the virus, the species, breed, and age of the infected animal; older animals are generally more susceptible [9]. The worldwide economic losses due to bluetongue have not been expressed in exact numbers, but the estimate is 3 billion US\$ a year [10]. The losses are both direct (death, abortions, weight loss, or reduced milk yield and meat efficiency) and, what is more important, indirect as a result of export restrictions for live animals, their semen, and some products such as fetal bovine serum.

Bluetongue virus is a non enveloped virus composed of three shells. The inner shell is composed of 120 copies of VP3 $(100 \mathrm{kDa})$ [11] and contains minor amounts of 3 enzymatic proteins involved in transcription and replication, namely, the RNA-dependent RNA polymerase VP1 $(149 \mathrm{kDa})$, the RNA capping enzyme VP4 $(76 \mathrm{kDa})$, and the dsRNA helicase VP6 $(36 \mathrm{kDa})$ that are located at the fivefold symmetry axis of the particle [12]. The middle shell is composed of 780 copies of VP7 $(38 \mathrm{kDa})$ that are arranged as 260 trimers [12, 13]. The outer shell is composed of two structural proteins: VP2 $(111 \mathrm{kDa})$ and VP5 $(59 \mathrm{kDa})$. A total of 180 molecules of VP2 are arranged as 60 surface spikes which are responsible for attaching the virus to the cell surface, whereas 360 molecules of VP5 form 120 globular-shaped structures that facilitate 
cell-membrane penetration [14-16]. Nonstructural proteins (NS1, NS2, NS3, NS3A, and NS4) probably participate in the control of BTV replication, maturation, and export from the infected cell. The NS1 protein was found to play role in viral morphogenesis $[17,18]$. The NS2 protein is the major component of viral inclusion bodies [17-20] and is also involved in recruitment of BTV mRNA for replication [21-23]. The NS3 protein acts as a viroporin, which enhances permeability of the cytoplasmic membrane and thus facilitates virus release from mammalian or insect cells [13, 23-25]. In addition, NS3 also allows BTV particles to leave host cells by a budding mechanism [26]. This probably operates in insect cells where no cytopathic effect is induced by BTV [20]. NS4 protein is expressed early after infection and localized in the nucleoli of BTV infected cells and plays an important role in virus-host interaction to counteract the antiviral response of the host [27].

Recent data obtained by cryoelectron microscopy of unfixed and unstained cores derived from BTV indicate that there are several unique features in the structure of the core [28]. The core has icosahedral symmetry and bristly appearance which is due to 260 prominent knob- or spikelike structures at all the local and strict threefold axes. Both biochemical and crystallographic analyses have been shown spikes to be trimers of VP7 [28-30]. The trimers are connected at a lower radius to a smooth layer of VP3 protein $[28,31]$. Recently it was reported that the two outer capsid proteins of BTV, VP2 and VP5, attach to the core, most likely involving VP7 $[32,33]$. Because of its multifarious roles, VP7 offers an interesting model to experimentally define intermolecular interactions in the BTV assembly process. Earlier it was reported that, in order to investigate the domains of VP7 that are involved in particle assembly, core-like particles (CLPs) containing VP7 and VP3 proteins were produced using multiple gene expression vectors [34]. Site-specific mutational studies of VP7 indicated that the intact carboxy terminus of the VP7 molecule is essential for particle formation but that the amino terminus can be modified by the addition of some foreign sequences without compromising the ability to form CLPs. Replacement of the single lysine residue of VP7 with leucine abrogated CLP formation, although replacement of either of the first two conserved cysteine residues (Cys-15 or Cys-65) [35-37] with serine did not prevent CLP formation. Based on this data we have made an attempt to find lead compounds that would modulate VP7 protein in abrogating CLP formation. Thus we have first carried out homology modeling of VP7 protein using reported X-ray structure from literature [38]. Mutational studies were carried out by constructing mutant model via single substitution at K255L and modeled. All the studies were carried out using DS. Novel virostatic compounds against BTV reported in literature $[39,40]$ were selected as ligands. Further docking with the selected ligands is done and the analysed results show the increased response of the ligands towards the K255L mutant models compared to VP7 model. Based on the high dock score, the compounds $\mathrm{T}-1, \mathrm{~T}-2$, and $\mathrm{T}-3$ were found to possess highest potentiality to act as potent leads in modulation of VP7 protein in abrogating CLP formation.

\section{Materials and Methodology}

2.1. Sequence Retrieval and Template Selection. The amino acid sequence of VP7 protein is retrieved in FASTA format from SWISS PROT database followed by BLAST against PDB for template selection. With known structures available in the $\mathrm{PDB}$, the BLAST is used to find the similarity of the sequence to closest homologous proteins and identifies the structure with high identity and similarity to be employed as template for homology modeling.

2.2. Sequence Alignment and Model Building. The sequence alignment process is carried out by extracting the sequence of template and aligned with the target sequence by using Clustal W. Using "MODELLER 9.11v" the 3D structure of VP7 is generated. Energy minimization of the modeled structure is carried out by applying CHARMm force fields and steepest descent algorithm followed by conjugant gradient algorithm in DS until the convergence gradient is satisfied.

2.3. K255L Mutant Construction. Mutations are substituted at single residue in the modeled VP7 protein and constructed the mutant model based on the wild type and energy minimization is carried out by applying CHARMm force fields.

\section{Model Validation}

3.1. Procheck. Procheck is used in validation of protein structure and models by verifying the parameters like Ramachandran plot quality, peptide bond planarity, bad nonbonded interactions, main chain hydrogen bond energy, C-alpha chirality and overall $\mathrm{G}$ factor, and the side chain parameters like standard deviations of chil gauche minus, trans and plus, and pooled standard deviations of chil with respect to refined structures.

3.2. Prosa. This program compares $Z$ scores between target and template structure. The $Z$ scores of model are a measure of compatibility between its sequence and structure. The model $Z$ score should be comparable to the $Z$ scores obtained from the template.

3.3. RMSD. Root Mean Squared Deviation (RMSD) is commonly used to represent the distance between two objects. This value indicates the degree to which two threedimensional structures are similar. The lower the value is, the more similar the structures are. SPDBV program was used in calculating the RMSD value between the template 1BVP and our model structure.

\section{Molecular Docking}

4.1. Ligand Generation and Optimization. Using Chemsketch the structure of the ligands taken for binding analysis is drawn. Further ligand preparation with constraint parameters such as tautomer and isomer generation and all the 


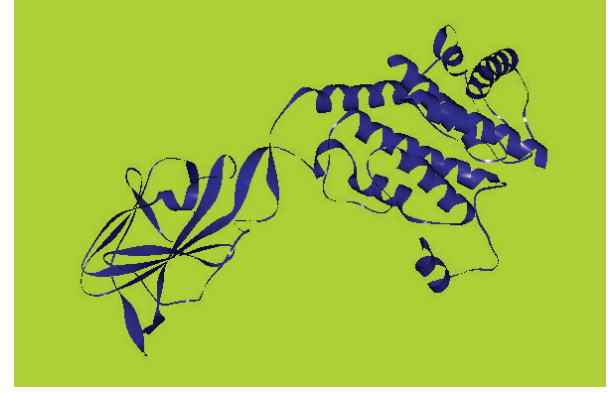

FIgURE 1: Modeled VP7 protein of BTV.

duplicate structures are removed. Catalyst algorithm in DS was used in 3D structure generation.

4.2. Active Site Identification. "Eraser" algorithm in DS was used in determining binding pockets of the receptor after energy minimization. The active site of the protein is first identified and it is defined as the binding site. This method employs a cavity detection algorithm for detecting invaginations in the protein as candidate active site regions.

4.3. Docking Studies. The docking method used in this study of docking of ligands into protein active site was LigandFit method. To perform docking in the DS, a protocol called "Dockligands" (LigandFit) is selected among those listed under receptor-ligand interaction protocol cluster. Each ligand compound is given as input in the parameter meant for "input ligands" and the protocol was run for each of the modulators selected for the study. A shape comparison filter is combined with a Monte Carlo conformational search for generating various conformations of ligand consistent with the active site shape. For evaluating protein-ligand interaction energies candidate poses are minimized in the context of the active site using a grid based method. The final energy refinement of the ligand pose (or) pose optimization in LigandFit occurs by Broyden-Fletcher-Goldfarb-Shanno (BFGS) method. Thus docking analysis of the taken compounds with VP7 protein is carried out to predict the binding affinities based on scoring functions such as LigScore and PLP, JAIN, PMF, and dock score and their relative stabilities are also evaluated using their binding affinities. The ligand binding energies are calculated based on the high dock score of best conformation.

\section{Results and Discussion}

5.1. Sequence Retrieval and Template Selection. The amino acid sequence of VP7 protein is taken from SWISSPROT database containing 349 residues with accession number: P69362, entry name: VP7_BTV11, and protein name: core protein VP7. The FASTA sequence of the protein (1-349) is retrieved and submitted to BLAST against PDB database for selection of homologous structures to be employed as template for homology modeling. The BLAST results yield X-ray structure of 1BVP from bluetongue virusserotype 10 having the highest sequence identity of $100 \%$ with a resolution of $2.60 \mathrm{~A}_{0}$. All the further procedures are carried out using MODELLER 9.11v.

5.2. Sequence Alignment and Model Building. A sequence alignment between the template-target pair is an essential input to a homology modeling program. The sequence of 1BVP is extracted and aligned with the VP7 protein of BTV using Clustal W. On the basis of this alignment as input, model of the VP7 is built using "MODELLER 9.11v." Ten molecular models of VP7 are generated. The refinement process is carried out using DS by applying CHARMm force field and steepest descent method is applied with 0.001 minimizing RMS gradient and 2000 minimizing steps followed by conjugant gradient method till it reaches the satisfactory results for minimization. The energy refinement method gives the best conformation to the model (Figure 1).

5.3. K255L Mutant Construction. The model of mutant VP7 protein with mutation in the region of K255L was generated with "Build Mutant" protocol and energy is minimized.

5.4. Model Validation. To evaluate the quality of our model, the final refined modeled structure of VP7 of BTV is analyzed by the Procheck, Prosa, and RMSD calculation.

5.5. Procheck. Using Procheck, the overall stereochemical quality of the modeled protein is evaluated by analyzing the overall residue by residue geometry and psi and phi torsion angles of Ramachandran plot showing residues with most favorable region (95\%), generously allowed region $(0.3 \%)$, additionally allowed region (4.3\%), and disallowed region $(0.3 \%)$ in comparison to $1 B V P$ template $(90.7 \%, 0.0 \%, 8.6 \%$, and $0.7 \%)$, respectively. The Ramachandran's map of the VP7 model and the template (1BVP) are shown in Figure 2 and the plot statistics are given in Table 1.

5.6. Prosa. Quality assessment of the model via Prosa revealed that the BTV model matched NMR region of the plot with $Z$ score $(-7.93)$ which is reliable to the $Z$ score of the template 1BVP (-7.87) (Figure 3 ). It signifies the quality of our model.

5.7. RMSD. The Root Mean Square Deviation (RMSD) between predicted VP7 of BTV model and template is $0.3 \AA$. The low RMSD between the target and template reflects the presence of strong homology.

5.8. Active Site Identification. Binding site analysis is done for the generated model. This was carried out using a tool panel "binding site." By applying CHARMm force field the receptor is analysed for the active sites from the "find sites from the receptor cavities." Site 1 is selected from the displayed sites.

5.9. Docking Studies. Ligand fit program (Accelrys Discovery Studio 2.5) was used to study the binding modes of the taken compounds in the active sites of modeled BTV and 
TABLE 1: The percentage of residues in the core region of the Ramachandran plot for the built BTV model and the template.

\begin{tabular}{lcccc}
\hline Structure & Core & Allowed & Generous & Disallowed \\
\hline 1BVP & 90.7 & 8.6 & 0.0 & 0.7 \\
BTV & 95.0 & 4.3 & 0.3 & 0.3 \\
\hline
\end{tabular}

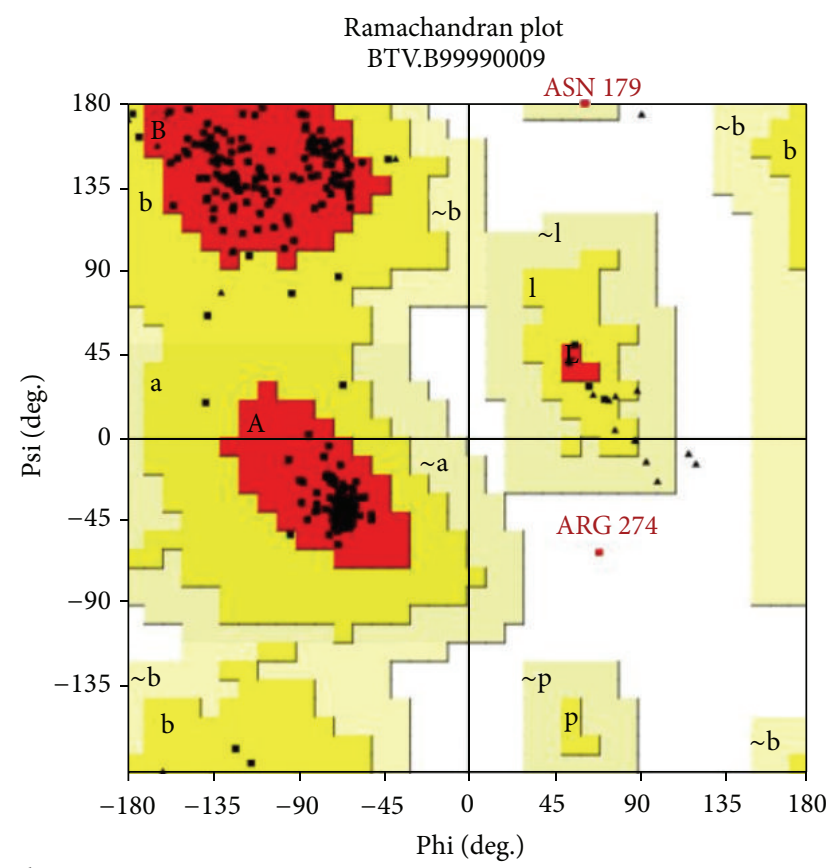

Plot statistics

Residues in most favored regions [A, B, L] $\quad 287 \quad 95.0 \%$

Residues in additional allowed regions $[\mathrm{a}, \mathrm{b}, \mathrm{l}, \mathrm{p}] \quad 13 \quad 4.3 \%$

Residues in generously allowed regions $[\sim \mathrm{a}, \sim \mathrm{b}, \sim 1, \sim \mathrm{p}] \quad 1 \quad 0.3 \%$

Residues in disallowed regions

Number of nonglycine and nonproline residues $\quad 302 \quad 100.0 \%$

Number of end residues (excl. Gly. and Pro.)

Number of glycine residues (shown as triangles)

Number of proline residues

Total number of residues

Based on an analysis of 118 structures of resolution of at least 2.0 angstroms and R-factor not greater than $20 \%$, a good quality model would be expected to have over $90 \%$ in the most favored regions.

(a)

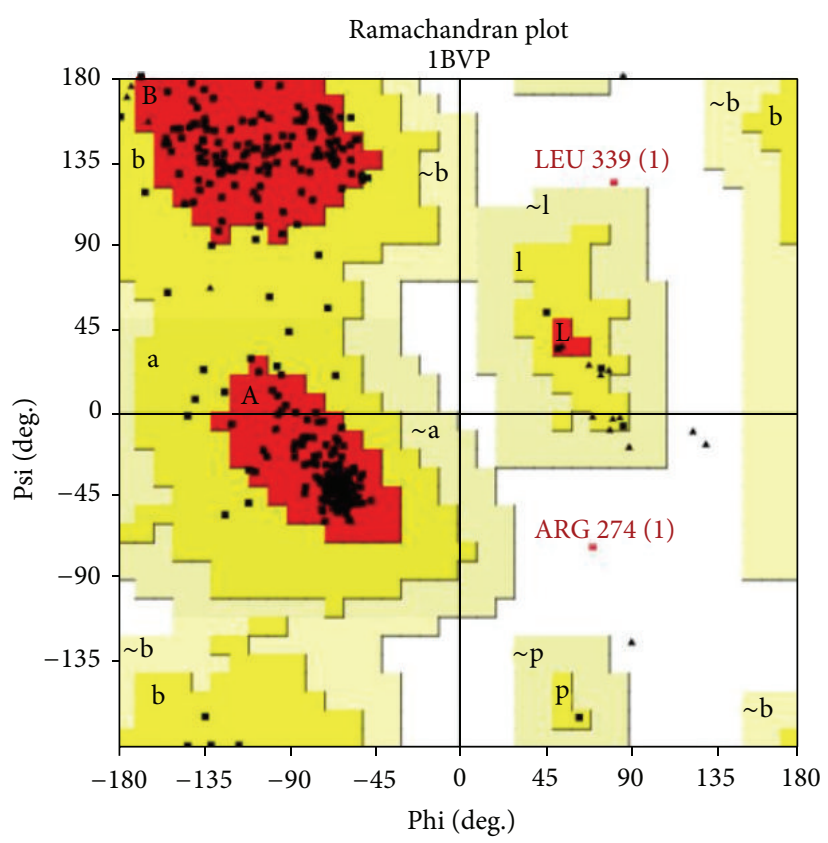

Plot statistics

Residues in most favored regions [A, B, L]

$274 \quad 90.7 \%$

Residues in additional allowed regions $[\mathrm{a}, \mathrm{b}, \mathrm{l}, \mathrm{p}]$

$26 \quad 8.6 \%$

Residues in generously allowed regions $[\sim \mathrm{a}, \sim \mathrm{b}, \sim \mathrm{l}, \sim \mathrm{p}] \quad 0 \quad 0.0 \%$

Residues in disallowed regions

Number of nonglycine and nonproline residues

- $2-0.7 \%$

$302 \quad 100.0 \%$

Number of end residues (excl. Gly. and Pro.)

Number of glycine residues (shown as triangles)

2

Number of proline residues

Total number of residues

23

21

Based on an analysis of 118 structures of resolution of at least 2.0 angstroms and R-factor not greater than $20 \%$, a good quality model would be expected to have over $90 \%$ in the most favored regions.

(b)

FIgURE 2: (a) Ramachandran's Map of VP7 protein of BTV and (b) Ramachandran's Map of 1BVP protein.

K255L mutant BTV. Dock score is used to estimate the ligandbinding energies. Apart from these, other input parameters for docking are also considered for evaluating the docking efficacy of proteins with ligands in our study. In this study, the ligands taken (Figure 4) are docked into the active site of BTV in order to find the receptor-ligand binding orientation, binding affinity, and binding-free energies. The docking run generated 10 poses for each of the analogs. Two important parameters have been considered for selecting potential compounds among the given input: (i) hydrogen bond details of the top-ranked pose and (ii) prediction of binding energy of the best docked pose using various scores calculated using Discovery Studio (LigScorel and 2, -PLP1 and 2, JAIN, PMF, and dock score) scores are taken for the analysis. The dock scores computed by the different scoring functions for these compounds are tabulated in Table 2. The dock score for all the docked ligands shows that T-1, T-2, and T-3 are having the highest dock scores. From the overall docking we identified 

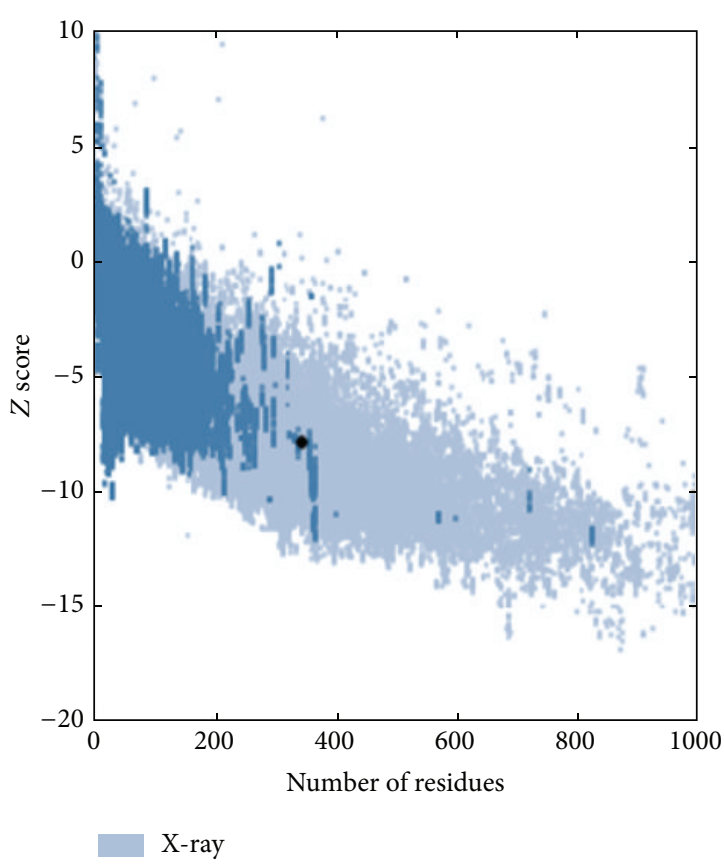

NMR

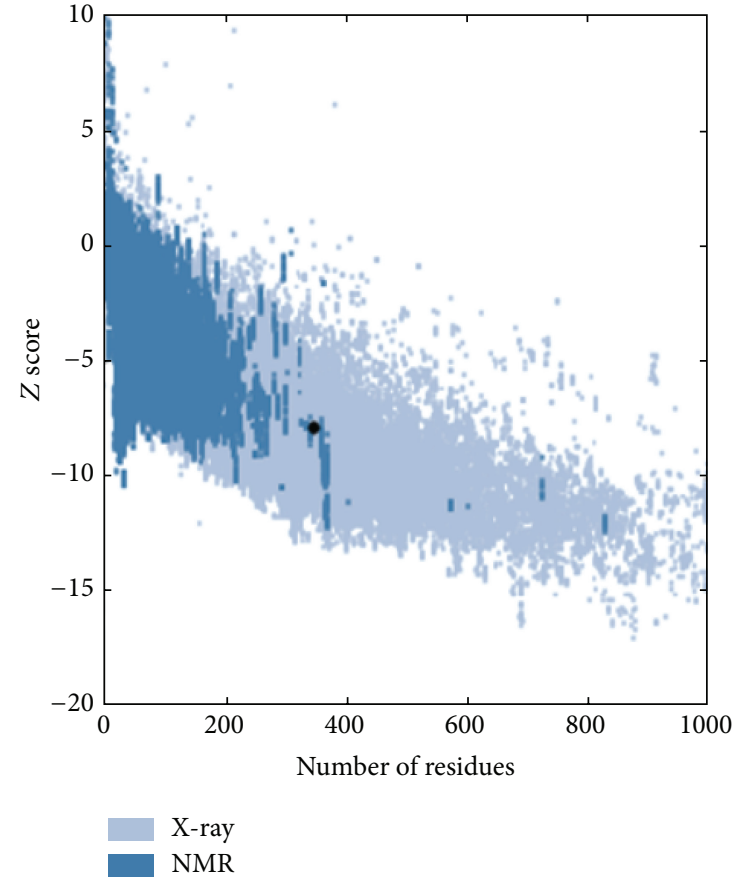

$Z$ score: -7.87

(b)

FIgURE 3: The plots of $Z$ Score values of VP7 of BTV and 1BVP, determined by NMR (shown in dark blue color) and by X-ray (shown in light blue color) using Prosa program. The black dots point out the $Z$ Scores of our model $(-7.93)$ and template $(-7.87)$, which indicates the reliability of the modeled VP7 protein of BTV.

TABLE 2: Dock scores of the ligand compounds into the modeled wild and mutant VP7 protein active site.

\begin{tabular}{lccccc}
\hline S. No. & Ligand & Wild & $\begin{array}{c}\text { Hydrogen } \\
\text { bonds }\end{array}$ & $\begin{array}{c}\text { Mutant } \\
\text { (K255L) }\end{array}$ & $\begin{array}{c}\text { Hydrogen } \\
\text { bonds }\end{array}$ \\
\hline 1 & T-1 & 41.38 & 1 & 54.47 & 5 \\
2 & T-2 & 35.23 & 2 & 55.42 & 4 \\
3 & T-3 & 34.44 & 2 & 52.53 & 7 \\
4 & T-4 & 38.40 & 3 & 43.57 & 3 \\
5 & T-7 & 38.34 & 3 & 31.48 & 3 \\
6 & T-8 & 11.60 & 1 & - & - \\
7 & T-9 & 11.832 & 1 & - & - \\
8 & T-10 & 13.20 & 1 & - & - \\
9 & T-1 3 & 34.23 & 1 & - & - \\
\hline
\end{tabular}

The stability of the best docked poses of T-1, T-2, and T-3 is evaluated by determining the hydrogen bonding between the receptor and ligand.

that T-2 is having a high dock score $55.42 \mathrm{~K} \cdot \mathrm{cal} / \mathrm{mol}$ with K255L mutant compared to that of the wild BTV.

\section{Conclusion}

In this study, we have predicted the structure for VP7 protein of BTV based on the template 1BVP and generated mutated model with single substitutions at K255L. Procheck, Prosa and RMSD calculation between the target and template proteins revealed the reliability of wild and mutant models for docking. Using LigandFit module of Discovery Studio software, docking studies were performed to investigate the role of ligand binding affinity at the active site of wild and mutant BTV. The results indicate that the mutant model may contribute to higher level binding interaction and binding affinity than wild model of BTV. Based on the scoring functions and individual residue interactions here we conclude that the compound $\mathrm{T}-2$ has more affinity at active site than others. The T-2 ligand with a dock score of 55.42 showed better results among all the taken compounds. Thus T-2 ligand could be good lead for development of novel virostatic agents in modulation of VP7 protein in abrogating CLP formation.

\section{Conflict of Interests}

The author declares that there is no conflict of interests regarding the publication of this paper.

\section{Acknowledgment}

The author acknowledges Professor V. Uma, Department of Chemistry, Nizam College, for providing technical assistance in carrying out this work. 


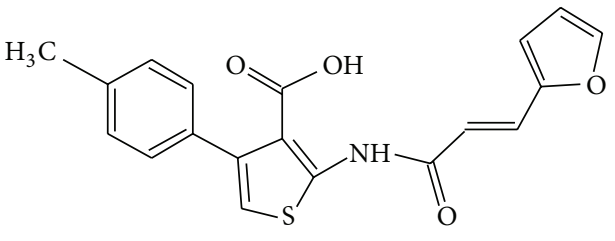

T-1<smiles>Cc1c(-c2nnc(-c3ccccc3)o2)[se]c2ncnc(Nc3ccc(Cl)cc3)c12</smiles>

T-3<smiles>Cc1c(-c2nnc(-c3ccc([N+](=O)[O-])cc3)o2)[se]c2ncnc(Nc3ccc(Cl)cc3)c12</smiles>

$\mathrm{T}-5$<smiles>Cc1c(-c2nnc(-c3ccc(Cl)cc3)o2)[se]c2ncnc(Nc3ccc(F)cc3)c12</smiles>

T-7<smiles>Cc1c(-c2nnc(-c3ccccc3)o2)[se]c2ncnc(Nc3ccc(F)c(Cl)c3)c12</smiles>

T-9<smiles>Cc1c(-c2nnc(-c3ccc([N+](=O)[O-])cc3)o2)[se]c2ncnc(Nc3ccc(F)c(Cl)c3)c12</smiles>

T-11<smiles>Cc1c(-c2nnc(-c3ccc(Cl)cc3)o2)[se]c2ncnc(Nc3ccc(F)cc3F)c12</smiles>

T-13<smiles>Cc1ccc(-c2csc(NC(=O)/C=C/c3cccs3)c2C(=O)O)cc1</smiles>

T-2<smiles>Cc1c(-c2nnc(-c3ccc(Cl)cc3)o2)[se]c2ncnc(Nc3ccc(Cl)cc3)c12</smiles>

T-4

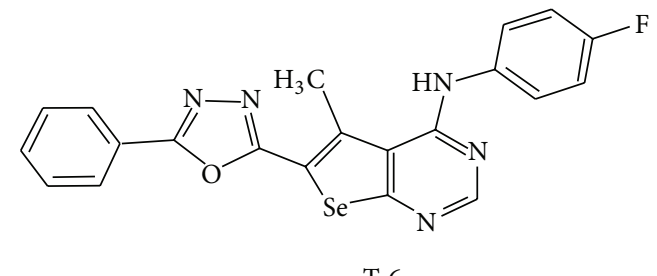

T-6<smiles>Cc1c(-c2nnc(-c3ccc([N+](=O)[O-])cc3)o2)[se]c2ncnc(Nc3ccc(F)cc3)c12</smiles>

T-8<smiles>Cc1c(-c2nnc(-c3ccc(Cl)cc3)o2)[se]c2ncnc(Nc3ccc(F)c(Cl)c3)c12</smiles>

$\mathrm{T}-10$

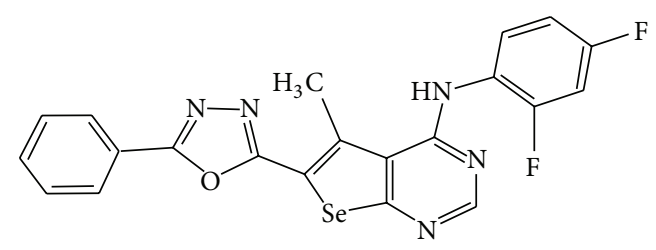

T-12<smiles>Cc1ccc(-c2csc(NC(=O)/C=C/c3ccccn3)c2C(=O)O)cc1</smiles>

T-14 


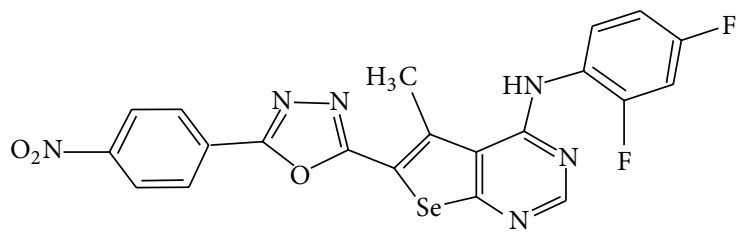

T-15

(b)

Figure 4: Ligands used for docking. (T-1) 2-\{[3-(2-furyl)acryloyl]amino\}-4-(4-methylphenyl)-3-thiophene carboxylic acid, (T-2) 2-\{[3(2-thiophenyl)acryloyl]amino\}-4-(4-methylphenyl)-3-thiophenecarboxylicacid, (T-3) N-(4-chlorophenyl)-5-methyl-6-(5-phenyl-1,3,4oxadiazol-2-yl)selenopheno[2,3-d]pyrimidin-4-amine, (T-4) N-(4-chlorophenyl)-6-(5-(4-chlorophenyl)-1,3,4-oxadiazol-2-yl)-5-methyl selenopheno[2,3-d]pyrimidin-4-amine, (T-5) N-(4-chlorophenyl)-5-methyl-6-(5-(4-nitrophenyl)-1,3,4-oxadiazol-2-yl)selenopheno[2,3d]pyrimidin-4-amine, (T-6) N-(4-fluorophenyl)-5-methyl-6-(5-phenyl-1,3,4-oxadiazol-2-yl)selenopheno[2,3-d]pyrimidin-4-amine, (T-7) 6-(5-(4-chlorophenyl)-1,3,4-oxadiazol-2-yl)-N-(4-fluorophenyl)-5-methylselenopheno[2,3-d]pyrimidin-4-nitrophenyl)-1,3,4-oxadiazol-2yl)selenopheno[2,3-d]pyrimidin-4-amine, (T-8) N-(4-Fluorophenyl)-5-methyl-6-(5-(4-nitrophenyl)-1,3,4-oxadiazol-2-yl)selenopheno[2,3d]pyrimidin-4-amine, (T-9) N-(3-chloro-4-fluorophenyl)-5-methyl-6-(5-phenyl-1,3,4-oxadiazol-2-yl)selenopheno[2,3-d]pyrimidin-4amine, (T-10) N-(3-chloro-4-fluorophenyl)-6-(5-(4-chlorophenyl)-1,3,4-oxadiazol-2-yl)-5-methylselenopheno[2,3-d]pyrimidin-4-amine, (T-11) N-(3-chloro-4-fluorophenyl)-5-methyl-6-(5-(4-nitrophenyl)-1,3,4-oxadiazol-2-yl)selenopheno[2,3-d]pyrimidin-4-amine, (T-12) $\mathrm{N}$-(2,4-difluorophenyl)-5-methyl-6-(5-phenyl-1,3,4-oxadiazol-2-yl)selenopheno[2,3-d]pyrimidin-4-amine, (T-13) 6-(5-(4-chlorophenyl)1,3,4-oxadiazol-2-yl)-N-(2,4-difluorophenyl)-5-methylselenopheno[2,3-d]pyrimidin-4-amine, (T-14) 2-\{[3-(2-pyridyl)acryloyl]amino\}4-(4-methylphenyl)-3-thiophene carboxylic acid, (T-15) N-(2,4-difluorophenyl)-5-methyl-6-(5-(4-nitrophenyl)-1,3,4-oxadiazol-2yl)selenopheno[2,3-d]pyrimidin-4-amine.

\section{References}

[1] A. J. Luedke, M. M. Jochim, and J. G. Bowne, "Preliminary bluetongue transmission with the sheep ked Melophagus ovinus (L.)," Canadian Journal of Comparative Medicine and Veterinary Science, vol. 29, no. 9, pp. 229-231, 1965.

[2] J. L. Stott, B. I. Osburn, and L. Alexander, "Ornithodoros coriaceus (pajaroello tick) as a vector of bluetongue virus," The American Journal of Veterinary Research, vol. 46, no. 5, pp. 11971199, 1985.

[3] C. Bouwknegt, P. A. van Rijn, J. J. M. Schipper et al., "Potential role of ticks as vectors of bluetongue virus," Experimental and Applied Acarology, vol. 52, no. 2, pp. 183-192, 2010.

[4] S. E. Brown, G. M. Gorman, R. B. Tesh, and D. L. Knudson, "Isolation of bluetongue and epizootic hemorrhagic disease viruses from mosquitoes collected in Indonesia," Veterinary Microbiology, vol. 32, no. 3-4, pp. 241-251, 1992.

[5] O. M. Radostits, D. C. Blood, and C. C. Gay, A Textbook of the Diseases of Cattle, Sheep, Pigs, Goats and Horses, VB Saunders, Philadelphia, Pa, USA, 8th edition, 1994.

[6] I. M. Parsonson, "Pathology and pathogenesis of bluetongue infections," Current Topics in Microbiology and Immunology, vol. 162, pp. 119-141, 1990.

[7] L. A. Wilbur, J. F. Evermann, R. L. Levings et al., "Abortion and death in pregnant bitches associated with a canine vaccine contaminated with bluetongue virus," Journal of the American Veterinary Medical Association, vol. 204, no. 11, pp. 1762-1765, 1994.

[8] J. F. Evermann, "Accidental introduction of viruses into companion animals by commercial vaccines," Veterinary Clinics of North America, vol. 38, no. 4, pp. 919-929, 2008.

[9] A. R. W. Elbers, A. Backx, K. Mintiens et al., "Field observations during the Bluetongue serotype 8 epidemic in 2006. II. Morbidity and mortality rate, case fatality and clinical recovery in sheep and cattle in the Netherlands," Preventive Veterinary Medicine, vol. 87, no. 1-2, pp. 31-40, 2008.
[10] W. J. Tabachnick, "The genetics of Culicoides variipennis and the epidemiology of bluetongue disease in North America," Annual Review of Entomology, vol. 45, pp. 20-40, 1996.

[11] J. M. Grimes, J. N. Burroughs, P. Gouet et al., "The atomic structure of the bluetongue virus core," Nature, vol. 395, no. 6701, pp. 470-478, 1998.

[12] E. L. Nason, R. Rothagel, S. K. Mukherjee et al., "Interactions between the inner and outer capsids of bluetongue virus," Journal of Virology, vol. 78, no. 15, pp. 8059-8067, 2004.

[13] P. Roy, "Bluetongue virus proteins," Journal of General Virology, vol. 73, no. 12, pp. 3051-3064, 1992.

[14] P. Roy and R. Noad, "Bluetongue virus assembly and morphogenesis," Current Topics in Microbiology and Immunology, vol. 309, pp. 87-116, 2006.

[15] M. Forzan, M. Marsh, and P. Roy, "Bluetongue virus entry into cells," Journal of Virology, vol. 81, no. 9, pp. 4819-4827, 2007.

[16] P. Roy, "Functional mapping of bluetongue virus proteins and their interactions with host proteins during virus replication," Cell Biochemistry and Biophysics, vol. 50, no. 3, pp. 143-157, 2008.

[17] S. M. Brookes, A. D. Hyatt, and B. T. Eaton, "Characterization of virus inclusion bodies in bluetongue virus-infected cells," Journal of General Virology, vol. 74, no. 3, pp. 525-530, 1993.

[18] R. J. Owens, C. Limn, and P. Roy, "Role of an arbovirus nonstructural protein in cellular pathogenesis and virus release," Journal of Virology, vol. 78, no. 12, pp. 6649-6656, 2004.

[19] K. Lymperopoulos, R. Noad, S. Tosi, S. Nethisinghe, I. Brierley, and P. Roy, "Specific binding of Bluetongue virus NS2 to different viral plus-strand RNAs," Virology, vol. 353, no. 1, pp. 17-26, 2006.

[20] I. Schwartz-Cornil, P. P. C. Mertens, V. Contreras et al., "Bluetongue virus: virology, pathogenesis and immunity," Veterinary Research, vol. 39, no. 5, article 46, 2008.

[21] A. Fukusho, Y. Yu, S. Yamaguchi, and P. Roy, "Completion of the sequence of bluetongue virus serotype 10 by the characterization of a structural protein, VP6, and a non-structural protein, 
NS2," Journal of General Virology, vol. 70, no. 7, pp. 1677-1689, 1989.

[22] A. K. Kar, B. Bhattacharya, and P. Roy, "Bluetongue virus RNA binding protein NS2 is a modulator of viral replication and assembly," BMC Molecular Biology, vol. 8, article 4, 2007.

[23] P. Roy and R. Noad, "Virus-like particles as a vaccine delivery system: myths and facts," Human Vaccines, vol. 4, no. 1, pp. 5-12, 2008.

[24] A. D. Hyatt, A. R. Gould, B. Coupar, and B. T. Eaton, "Localization of the non-structural protein NS3 in bluetongue virusinfected cells," Journal of General Virology, vol. 72, no. 9, pp. 2263-2267, 1991.

[25] Z. Han and R. N. Harty, "The NS3 protein of bluetongue virus exhibits viroporin-like properties," Journal of Biological Chemistry, vol. 279, no. 41, pp. 43092-43097, 2004.

[26] C. Wirblich, B. Bhattacharya, and P. Roy, "Nonstructural protein 3 of bluetongue virus assists virus release by recruiting ESCRT-I protein Tsg101," Journal of Virology, vol. 80, no. 1, pp. 460-473, 2006.

[27] M. Ratinier, M. Caporale, M. Golder et al., "Identification and characterization of a novel non-structural protein of bluetongue virus," PLoS Pathogens, vol. 7, no. 12, Article ID e1002477, 2011.

[28] B. V. V. Prasad, S. Yamaguchi, and P. Roy, "Three-dimensional structure of single-shelled bluetongue virus," Journal of Virology, vol. 66, no. 4, pp. 2135-2142, 1992.

[29] A. K. Basak, D. I. Stuart, and P. Roy, "Preliminary crystallographic study of bluetongue virus capsid protein, VP7," Journal of Molecular Biology, vol. 228, no. 2, pp. 687-689, 1992.

[30] E. A. Hewat, T. F. Booth, P. T. Loudon, and P. Roy, "Threedimensional reconstruction of baculovirus expressed bluetongue virus core-like particles by cryo-electron microscopy," Virology, vol. 189, no. 1, pp. 10-20, 1992.

[31] P. T. Loudon and P. Roy, "Assembly of five bluetongue virus proteins expressed by recombinant baculoviruses: inclusion of the largest protein VP1 in the core and virus-like particles," Virology, vol. 180, no. 2, pp. 798-802, 1991.

[32] E. A. Hewat, T. F. Booth, and P. Roy, "Structures of bluetongue virus particles by cryoelectron microscopy," Journal of Structural Biology, vol. 109, no. 1, pp. 61-69, 1992.

[33] H. M. Liu, T. F. Booth, and P. Roy, "Interactions between bluetongue virus core and capsid proteins translated in vitro," Journal of General Virology, vol. 73, no. 10, pp. 2577-2584, 1992.

[34] H. le Blois and P. Roy, "A single point mutation in the VP7 major core protein of bluetongue virus prevents the formation of corelike particles," Journal of Virology, vol. 67, no. 1, pp. 353-359, 1993.

[35] T. F. Kowalik and J. K.-K. Li, "Sequence analyses and structural predictions of double-stranded RNA segment S1 and VP7 from United States prototype bluetongue virus serotypes 13 and 10," Virology, vol. 172, no. 1, pp. 189-195, 1989.

[36] T. F. Kowalik and J. K.-K. Li, "Bluetongue virus evolution: sequence analyses of the genomic S1 segments and major core protein VP7," Virology, vol. 181, no. 2, pp. 749-755, 1991.

[37] C. Prehaud, K. Takehara, A. Flamand, and D. H. L. Bishop, "Immunogenic and protective properties of rabies virus glycoprotein expressed by baculovirus vectors," Virology, vol. 173, no. 2, pp. 390-399, 1989.

[38] J. Grimes, A. K. Basak, P. Roy, and D. Stuart, "The crystal structure of bluetongue virus VP7," Nature, vol. 373 , no. 6510, pp. 167-170, 1995.
[39] L. Gu, V. Musiienko, Z. Bai, A. Qin, S. W. Schneller et al., "Novel virostatic agents against bluetongue virus," PLOS ONE, vol. 7, Article ID e43341, 2012.

[40] Y. Kotaiaha, N. Harikrishnaa, K. Nagarajua, D. V. R. Sai Gopalb, V. Taranathb, and C. Venkata Raoa, "Synthesis and antiviral activity of 1, 3, 4-oxadiazolyl selenopheno[2, 3-d]pyrimidines as novel bluetongue virus inhibitors," Journal of Chemical and Pharmaceutical Research, vol. 5, pp. 359-369, 2013. 

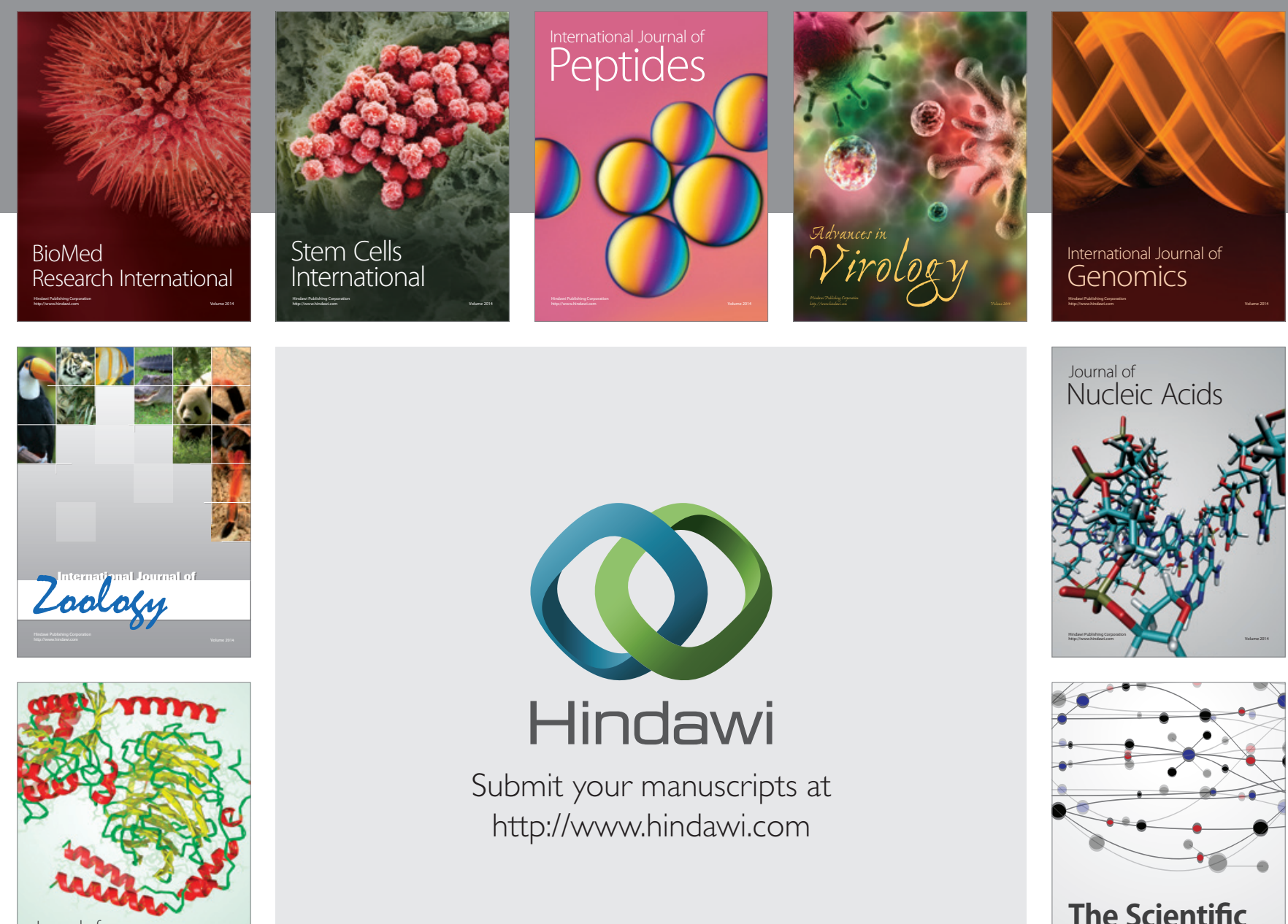

Submit your manuscripts at

http://www.hindawi.com

Journal of
Signal Transduction
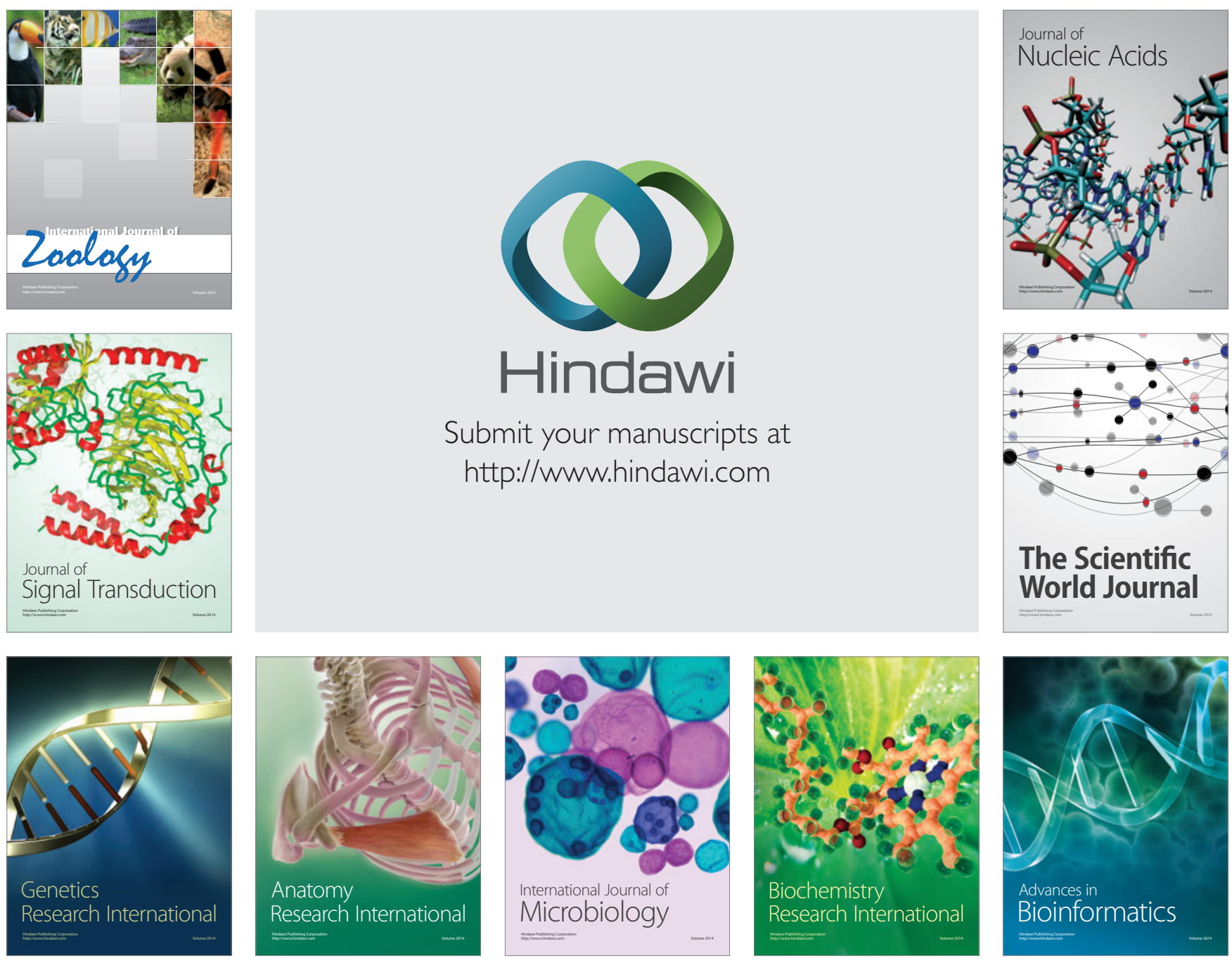

The Scientific World Journal
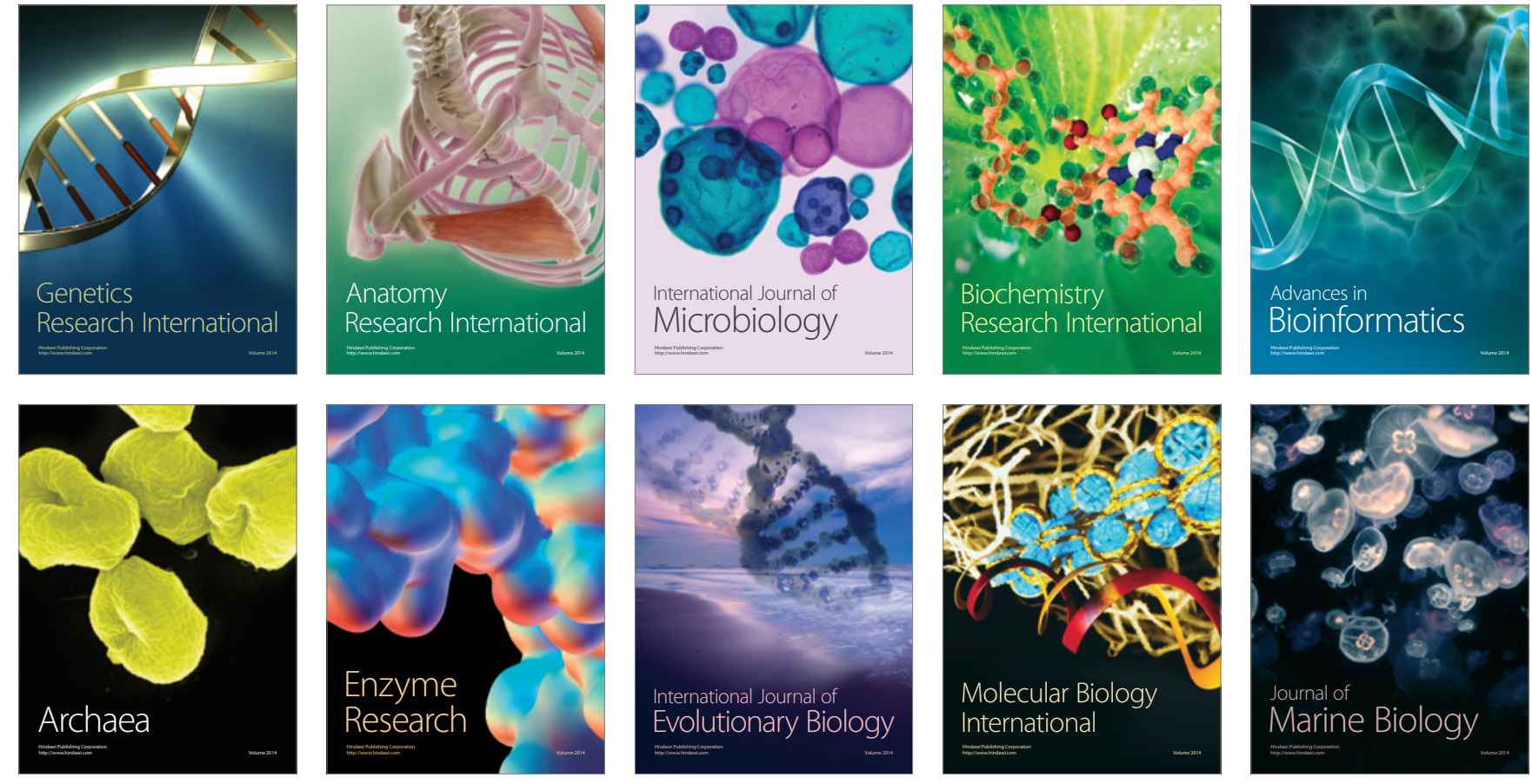